\title{
9th Tuscany Retreat on Cancer Research: genetic profiling, resistance mechanisms and novel treatment concepts in cancer
}

\author{
L Peintner ${ }^{1}$, F Novelli ${ }^{2}$, A Smirnov ${ }^{2}$, U Maurer ${ }^{1,3}$, C Borner ${ }^{1,3}$ and S von Karstedt ${ }^{\star, 4}$ \\ Cell Death and Differentiation (2016) 23, 183-184; doi:10.1038/cdd.2015.148; published online 13 November 2015
}

9th Tuscany Retreat on Cancer Research, Palazzo di Piero, Chiusi, Tuscany, Italy, 1-8 August 2015

Every 2 years, an international assembly of scientists meet in the same fifteenth-century castle in Tuscany with the aim of better understanding cancer and its resistance to therapy.

The meeting's opening plenary lecture was given by Kevin Ryan (The Beatson Institute, UK), who presented that absence of a lysosomal glycosidase leads to craniofacial deformations, behavioral abnormalities and the accumulation of cleaved LC3. He proposed that inappropriate glycan breakdown causes a bottleneck in autophagic flux resulting in a retardation of autophagy. Elzbieta Kania (The Polish Academy of Sciences, Poland) presented data on the role of autophagy and apoptosis downstream of Verapamil.

Next, the laboratory of Christoph Borner (University of Freiburg, Germany), the main meeting organizer, showed that cells infected by the Semliki Forest virus undergo apoptosis in a Bax/Bak-independent manner, but through MAVS-mediated recruitment of caspase-8 to the mitochondria. Christoph Borner's PhD students and Postdocs Simon Neumann, Lukas Peintner, Florian Haun and Laura Faletti presented their insights into complex formation and posttranslational modifications of PUMA, how the fungal protein gliotoxin signals to BIM phosphorylation to induce anoikis and how TNF sensitizes to FasL-induced apoptosis.

Ulrich Maurer reported his recent research on phosphorylation of Tip60 on S90 and its role in regulation of transcription. His student Florian Preiss addressed the search for a GSK-3regulated transcription factor that induces PUMA upon growth factor withdrawal and Lisa Schlicher investigated the role of phosphorylation of CYLD in the regulation of TNF-induced cell death.

Beatrice Macchi (University of Rome 'Tor Vergata', Italy) presented that targeting glucose uptake in cancer cells by an amino sugar had a strong effect on the induction of autophagy and apoptosis, and Antonio Mastino and Emanuela Papaianni
(University of Messina, Italy) showed data on the mechanism of how HPV viruses form in autophagosomal vesicles.

The work of Dagmar Kulms (University of Dresden, Germany) revealed that in skin cells, UVB exposure and mutated p53 cooperate to induce high levels of TNF. TNF eliminated premalignant cells before further DNA damage could accumulate and initiate carcinogenesis. Thereby, she proposed a tumor-suppressive function for mutated p53 during early carcinogenesis, a concept that was intensively discussed while sunbathing.

Gerry Melino (Leicester, UK/Rome, Italy) presented a molecular dynamic model of p53 implying that it follows an induced-fit conformational change that stabilizes the DNA-bound tetramer. His student Flavia Novelli identified mutation-specific siRNAs for the therapeutic silencing of a p63 missense mutation that causes ectodermal dysplasia. Artem Smirnov from Melino's laboratory identified FOXM1 as an indirect p63 target that prevents cell death. Ivano Amelio demonstrated that TAp73 represses angiogenesis through degradation of HIF1a. Varvara Petrova then showed that TAp73 transcriptionally represses the BNIP3 gene overexpressed in lung cancer. Massimiliano Agostini (University of Rome, Italy) reported that the new p63 target gene, $Z N F 750$, is involved in proliferation and cell migration independently of EMT, and Alberto Marini (Leicester, UK) presented that overexpression of the long non-coding RNA uc. 63 is associated with poor prognosis in patients and that its knockdown induces apoptosis.

The group of Mavash Tavassoli (Kings College London, UK) including Nina Raulf and Hersi Hersi presented that caspase-8 and annexin-A1 are potential biomarkers for the sensitivity of head and neck cancer cells to TRAIL and SMAC mimetic treatment. In addition, the laboratory also investigates

\footnotetext{
${ }^{1}$ Institute of Molecular Medicine and Cell Research, Albert Ludwigs University of Freiburg, Stefan Meier Strasse 17, Freiburg D-79104, Germany; ${ }^{2}$ Department of Experimental Medicine and Surgery, University of Rome 'Tor Vergata', Rome 00133, Italy; ${ }^{3}$ Spemann Graduate School of Biology and Medicine, Albert Ludwigs University of Freiburg, Albertstrasse 19a, Freiburg D-79104, Germany and ${ }^{4}$ Centre for Cell Death, Cancer and Inflammation, UCL Cancer Institute, University College London, 72 Huntley Street, London WC1E 6DD, UK

*Corresponding author: S von Karstedt, Centre for Cell Death, Cancer and Inflammation, UCL Cancer Institute, 72 Huntley Street, London WC1E 6DD, UK. Tel: +44 207679 6261; Fax: +44 207679 6925; E-mail: s.karstedt@ucl.ac.uk
} 
circulating RNA markers that can be diagnosed by analyzing peripheral blood.

Heike Bantel (Hannover Medical School, Germany) showed that TRAIL/Bortezomib treatment is effective in killing hepatocellular carcinoma cells selectively. Karin Nowikovsky (Medical University of Vienna, Austria) presented that the gallium complex KP46 decreases intracellular iron levels, which leads to an induction of p53.

Henning Walczak (UCL Cancer Institute, UK) presented that absence of HOIP, the central catalytically active component of the LUBAC, causes embryonic lethality. Silvia von Karstedt demonstrated that KRAS-mutated cancers are promoted by tumor cell-expressed murine TRAIL-R through Rac1 activation. Annalisa Conti then went on to show that premalignant cells expressing oncogenic KRAS are sensitized to apoptotic camptothecin/SMAC mimetic treatment through NOXA upregulation. Antonella Montinaro showed that smallmolecule CDK9 inhibition combined with TRAIL treatment prolonged survival of mice with lung cancer.

That cancer cells depend on the interaction between ErbB, Ras and Nucleolin was shown by Ronit Pinkas-Kramarski (University of Tel-Aviv, Israel) and her students Eran Schmukler and Eya Wolfson. Ras inhibition enhanced autophagy, which partially protected cells from death. Assaf Menachem and Or Bodner, representing the laboratory of Yoel Kloog (University of Tel-Aviv, Israel), showed that small RNAs could be transferred from astrocytes to lung tumor cells through GAP junctions, resulting in their protection from chemotherapy-induced cell death, and treatment with a Ras inhibitor and a galectin-3 inhibitor inhibited growth of anaplastic thyroid cancer.

Thomas Grünewald (Ludwigs Maximilians University, Germany) presented that Ewing Sarcoma is driven by a genetic fusion of EWSR1 to FLI1 binding to GGAA microsatellites and forming a new enhancer for SNP-prone oncogenes such as EGR2 and ADO.

Scott Lowe (Memorial Sloan Kettering Cancer Center, US) presented that MAPK and PI3K undergo compensatory feedback upregulation upon treatment with Trametinib. Moreover, he showed that reinstalling APC expression in colon polyps that develop as a result of APC deletion leads to their disappearance. These data strongly suggest that reinstalling the WNT pathway might be an effective therapeutic strategy for colon carcinoma.

Mads Daugaard (Vancouver Prostate Centre, Canada) and his postdoc Nader Al Nakouzi showed that VAR2CSA, a protein expressed by the malaria pathogen Plasmodium, binds to chondroitin sulfate on proteoglycans highly expressed by cancer cells. Therefore, conjugating drug molecules to recombinant rVAR2 might be a novel strategy to deliver drugs selectively to a broad range of cancers.

Reuven Stein (Tel Aviv University, Israel) and his students Bar Ben Baruch and Alina Bespalto demonstrated that pharmacological inhibition of CD38 delayed cancer progression by decreasing NFKB signaling. Liora Lindenboim and Lotan Levin focused on the effect of Bax on the nucleus and the LINC complex.

Barak Rotblat (Ben Gurion University of the Negev, Israel, special young investigator seminar) showed that a p73 antisense RNA (LncRNA p73-AS1) is associated with poor prognosis in glioblastoma and promotes colony formation and migration.

Poul Sorensen (University of British Columbia, Canada) demonstrated that, while usually cells undergo senescence once they are exposed to hypoxia or starvation, a mutation in the kinase EF2K is able to block this effect.

Next, Michael Bergmann and his student Johannes Längle (Medical University of Vienna, Austria) showed data on the role of LINE1 in maintaining telomere length by enhancing expression of the shelterin complex and hTERT.

Rudolf Oehler and his student Katharina Strasser (Medical University of Vienna, Austria) presented that the complement component $\mathrm{C} 1 \mathrm{q}$ binds apoptotic cells, thereby attracting cell-removing macrophages. In cancer tissue, cell removal is impaired. Therefore, it is tempting to speculate whether this might contribute to cancer-associated inflammation. Christine Brostjan (Medical University of Vienna, Austria) showed that intermediate CD14- and CD16-expressing monocytes are attracted by early-stage but not metastatic cancer cells. Balazs Hegedues (Medical University of Vienna, Austria) revealed that hTERT and ITGA7 might represent 'driver-like' mutations in mesothelioma.

After 6 days of scientific discussions, wine tasting, beer consumption, nocturnal jam sessions and chronic sleep deprivation, the meeting drew to a close. Once again, this meeting has been a fantastic week of science to be continued in 2 years.

\section{Conflict of Interest}

The authors declare no conflict of interest.

Acknowledgements. We would like to thank our sponsors in no particular order: The Spemann Graduate School of Biology and Medicine, Cell Death \& Differentiation, Roche, Kairos Therapeutics, Chemometec, Tel Aviv University and the Medical University of Vienna. 